\title{
Improved Iterative Prediction Reconstruction for Compressive Whiskbroom Imaging
}

\author{
Yingbiao Jia ${ }^{1, a}$, Zhongliang Luo ${ }^{1, b}$ \\ ${ }^{1}$ School of Information Science and Engineering, Shaoguan University, Shaoguan, China

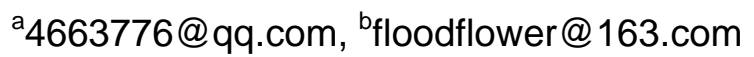

\begin{abstract}
Keywords: Hyperspectral Remote Sensing, Whiskbroom Compressive imaging, Reconstruction, Multi-Task Compressive Sensing, Iterative

Abstract: Compressive Sampling is suitable for remote hyperspectral imaging, as it can simplify the architecture of the onboard sensors. The reconstruction process is an indispensable component of the hyperspectral imaging as it decodes the compressive measurements to render a three-dimensional spatio-spectral estimate of the scene. The existed reconstruction methods mainly concentrated in reducing the algorithm complexity and increasing the reconstruction accuracy, not taking into account the sensing paradigm of the onboard sensors, such as compressive whiskbroom imaging. For this reason, an improved iterative prediction reconstruction algorithm employing Multi-Task Compressive Sensing for compressive whiskbroom imaging is proposed. Experimental results run on raw data from AVIRIS confirm the validity of the proposed method.
\end{abstract}

\section{Introduction}

Compressive Sampling $(\mathrm{CS})^{[1]}$ is a new signal-acquisition paradigm, which shows that a sparse or compressible signal can be recovered from a relatively small number of linear measurements(random projections). Therefore, the acquisition presents appealing properties such as simple sampling complexity, especially in the applications of resource-constrained, such as the remote acquisition of hyperspectral imagery for space-borne and air-borne earth observation.

In the hyperspectral remote sensing, the image is captured using a range of spectra from ultraviolet to visible to infrared, and a three-dimensional spatio-spectral data of a scene is created using light from different parts of the spectrum. It is costly for imaging at wavelength outside the visible light, where manufacturing detectors is very expensive. CS could be used to design cheaper sensors, or sensors providing better resolution for an equal number of detectors. As it is costly to acquire each pixel of the hyperspectral imagery, it becomes very attractive to use the CS approach to acquire hyperspectral data, which has been called hyperspectral compressive imaging. Hyperspectral compressive imaging allow to design sensors requiring a smaller memory buffer, fewer detectors, and a reduced volume of data to transmit.

The reconstruction process is an indispensable component of the hyperspectral compressive imaging as it decodes the CS measurements to render a three-dimensional spatio-spectral estimate of the scene. The application of CS theory to hyperspectral image acquisition is not straightforward mainly due to the complexity and accuracy of the reconstruction stage. Several strategies for reconstructing the HSI data from these compressive measurements have been proposed, and the reconstruction quality can be improved from intelligent use of prior knowledge about the hyperspectral data, such as the redundancy of hyperspectral images in both the spatial and spectral dimension. See for example [2], where hyperspectral images is decoded from compressive samples by exploiting its spatial 2D piecewise smoothness, low-rank property and adjacent spectrum correlation. See [3] for an overview of hyperspectral compressive imaging. In a word, the existed reconstruction methods mainly concentrated in reducing the algorithm complexity and increasing the reconstruction accuracy, not taking into account the sensing paradigm of the onboard sensors. In the moving airplane and satellite platforms, compressive hyperspectral imaging need to design compressive sensor based on pushbroom and whiskbroom scanning ${ }^{[4]}$.The reconstruction of compressive whiskbroom imaging can be regarded as a multivector CS-recovery problem, so it can be solved with the existed CS reconstruction algorithms, such as the Multi-Task Bayesian 
Compressive Sensing (MT-BCS) $)^{[5]}$, which introduces a hierarchical Bayesian framework into the CS-recovery problem to share prior information across the multiple vectors.

In this paper an improved reconstruction algorithm based on MT-BCS is proposed. we consider the compressive whiskbroom imaging and investigate suitable reconstruction algorithms that are able to take advantage of correlations in the spatial dimensions. The proposed algorithm requires separately sensed spectral vectors, which is compatible with the structure of whiskbroom sensors. We show that the iterative prediction method is effective at achieving a reconstruction quality better than that obtained by the conventional approach.

\section{Multi-Task Bayesian Compressive Sensing}

CS is a framework whereby one performs linear measurements $\boldsymbol{y} \in \mathbb{R}^{\mathrm{M}}$ to reconstruct a vector $\boldsymbol{x} \in \mathbb{R}^{N}$, with the assumption that $x$ is sparse in the basis represented by the matrix $\boldsymbol{\Psi} \in \mathbb{R}^{N \times N}$. The mapping $\boldsymbol{y} \rightarrow \boldsymbol{x}$ constitutes an inverse problem, often solved using $l_{1}$ regularization or related techniques. Compressive sensing has been considered from a Bayesian perspective in [6] and the reconstruction problem in CS can be solved more effectively by utilizing the relevance vector machine from the sparse Bayesian learning literature.

In the multivector CS-recovery research, if $L>1$ sets of compressive measurements $\left\{y_{i}\right\}_{i=1, \cdots, L}$ are performed, each of the associated $\left\{\boldsymbol{x}_{i}\right\}_{i=1, \cdots, L}$ can be recovered independently at a time. The mapping $\boldsymbol{y}_{i} \rightarrow \boldsymbol{x}_{i}$ is an independent CS recovery problem, which can be framed as a sparse linear-regression problem and solved by a Bayesian algorithm. In many applications the L "tasks" defined by the mappings $\boldsymbol{y}_{i} \rightarrow \boldsymbol{x}_{i}$ are not statistically independent, and it may be possible to improve the performance of the inversion if statistical inter-relationships are exploited. MT-BCS address this problem within a multi-task learning setting, wherein the mapping $\boldsymbol{y}_{\boldsymbol{i}} \rightarrow \boldsymbol{x}_{i}$ for each task corresponds to inferring the parameters (such as wavelet coefficients) associated with the desired signal, and a shared prior is placed across all of the L tasks. Under this hierarchical Bayesian modeling, data from all L tasks contribute toward inferring a posterior on the hyperparameters, and once the shared prior is thereby inferred, the data from each of the $\mathrm{L}$ individual tasks is then employed to estimate the task-dependent wavelet coefficients.

\section{Proposed Technique}

We propose a new reconstruction algorithm for compressive whiskbroom imaging, in which each spectral vectors is acquired with separate random projections, and the new algorithm reconstruct the entire data cube capturing the correlations in both spatial and spectral directions.

The whiskbroom scan, which is built on the imaging of a single pixel, or spatial location, at a time, is a widely used scanning paradigm in hyperspectral remote sensing. A whiskbroom architecture for the compressive acquisition of hyperspectral imagery is depicted conceptually in [4].

We represent hyperspectral images $\mathrm{X} \in \mathbb{R}^{N_{l} \times N_{p} \times N_{\lambda}}$ as a $3 \mathrm{D}$ collection of samples, where $N_{l}$ and $N_{p}$ represent spatial dimensions and $N_{\lambda}$ represents the spectral dimension. Hence, $\boldsymbol{X}$ can be considered as a collection of $N_{l} \times N_{p}$ spectral vectors $\boldsymbol{X}_{i, j,:} \in \mathbb{R}^{N_{\lambda}}\left(i=1, \cdots, N_{l} ; j=1, \cdots, N_{p}\right)$. We refer to this configuration as $N_{\lambda}-N_{l} \times N_{p}$ cube.

For what concerns the acquisition of the hyperspectral whiskbroom compressive imaging, it consists in the collection of the measurements for each spectral vector $\boldsymbol{X}_{i, j, .}$. The measurement process of each spectral vector is $\boldsymbol{Y}_{i, j,:}=\boldsymbol{\Phi}_{i, j} \boldsymbol{X}_{i, j,:}$, where $\boldsymbol{Y}_{i, j,:} \in R^{N_{m}}$, and $\Phi_{i, j}$ is of size $N_{m} \times N_{\lambda}$. Here each sensing matrix $\Phi$ is taken as Gaussian i.i.d. and $N_{m}<N_{\lambda}$. For simplicity, the same value $N_{m}$ is taken for all spectral vectors.

The measurements of all spectral vectors are then collected in the matrix $\boldsymbol{Y}$. The compressive acquisition protocol of the whole hyperspectral data is $\boldsymbol{Y}=\Gamma(\boldsymbol{X})$, where $\Gamma: \mathbb{R}^{N_{1} \times N_{p} \times N_{\lambda}} \rightarrow \mathbb{R}^{N_{1} \times N_{p} \times N_{m}}$ is determined by the compressive sensor. It is easy to separate reconstruction each spectral vector using 
common CS reconstruction algorithm. However, the separate reconstruction does not yield a sufficiently accurate estimate of the original hyperspectral images, since it lacks exploiting spatial correlation. As a matter of fact, this inaccurate reconstruction will be the initialization point of our proposed reconstruction algorithm.

The main concept behind our new reconstruction algorithm is to exploit correlation among the spatial directions by iteratively predicting each spectral vector and reconstructing the prediction error only, which is more compressible than the spectral vector itself. If the prediction filter is accurate, the prediction error is expected to be more compressible than the full signal, and the reconstruction will yield better results ${ }^{[7]}$.If a prediction of the hyperspectral data $\mathbf{X}_{i, j,:}$ is obtained, e.g., applying the operator $\mathrm{P}(\cdot, \cdot)$ to vectors $\mathbf{X}_{i-1, j,:}$ and $\mathbf{x}_{i, j-1, ;}$ of some initial reconstruction, then we can cancel out the contribution of this predictor from the measurements of $\mathbf{X}_{i, j,:}$, and reconstruct only the prediction error instead of the full spectral vector.

Prediction/reconstruction techniques have also been considered in [8,9] for different applications. In particular, the iterative procedure starts from the initial reconstruction $\mathbf{X}_{i, j,:}^{(0)}$ of all spectral vectors. The initial reconstruction can be solved with the well known $l_{1}$-norm convex regularizer algorithm:

$$
\mathbf{X}_{i, j,:}^{(0)} \leftarrow \arg \min \left\|\boldsymbol{\Psi} \mathbf{X}_{i, j,:}\right\|_{1} \text { s.t. } \quad \boldsymbol{\Phi}_{i, j} \mathbf{X}_{i, j,:}=\mathbf{Y}_{i, j,:}
$$

where $\boldsymbol{\Psi}$ can represent a wavelet basis, with the wavelet decomposition the wavelet coefficients are sparse.

In this paper, for every spectral vector we obtain its prediction from the adjacent spectral vectors at previous iteration. For the sake of simplicity, we take the mean data of the left front vector and left upper vector. The proposed iterative reconstruction algorithm is shown here:

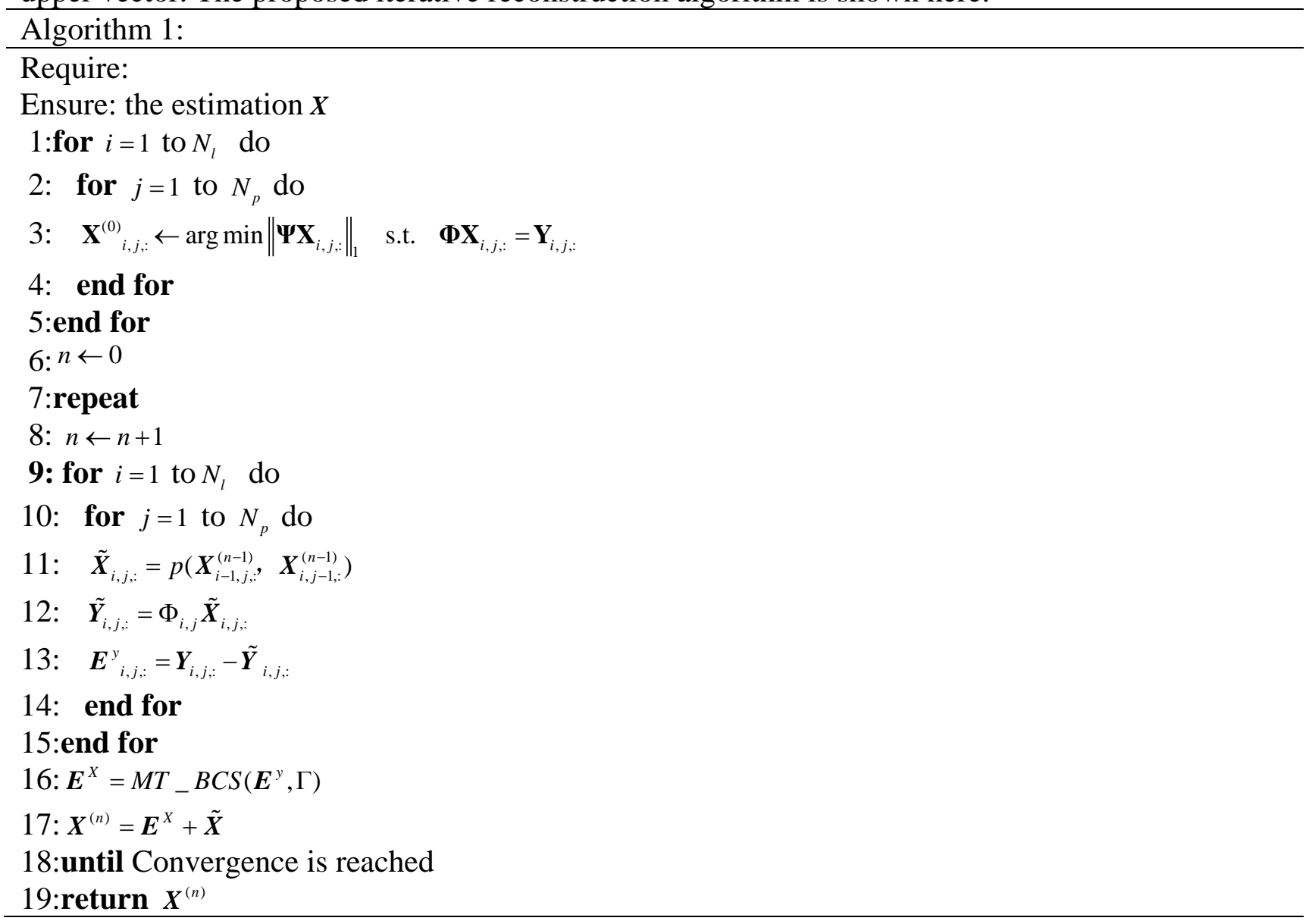




\section{Experimental Results}

Traditional hyperspectral sensors that use whiskbroom scanning include AVIRIS, which is a spectrometer with 224 bands, and the size of the image is 512 lines and 680 pixels. Our simulations are mainly based on the scenes of Cuprite from AVIRIS. The corresponding hyperspectral images is cropped to have the spatial resolution $256 \times 256$, and in addition $B=180$ spectral bands is selected after discarding water absorption bands.

As a primary measure of reconstruction quality, we calculate SNR averaged over all the bands under consideration. we compare our method with two other methods: the 11 algorithm separately applied on each spectral vector, and the MT-BCS method proposed in [5].The SNR performances at different measurement rate for the test data are shown in Figure 1. It can be observed that the SNR of the proposed method significantly outperforms the other two methods.
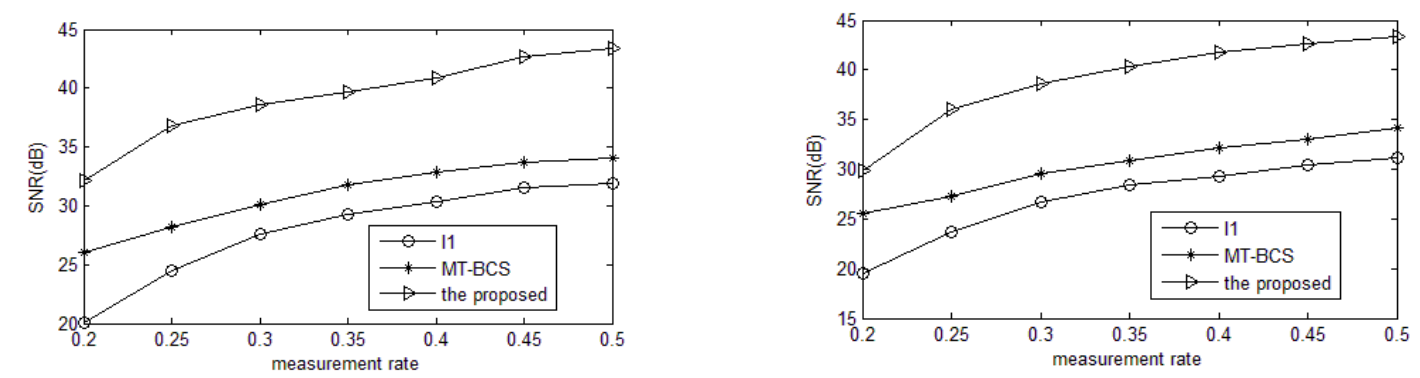

Fig. 1 Comparison of SNR performance (left:The first scene of Cuprite, right:The second scene of Cuprite)

Figure 2 shows the visual quality of the reconstructed images using three different methods when measurement rate is 0.1 . Obviously, the proposed method preserves image semantics much better than the image recovered by the other two methods via recovering edges and textures more faithfully.
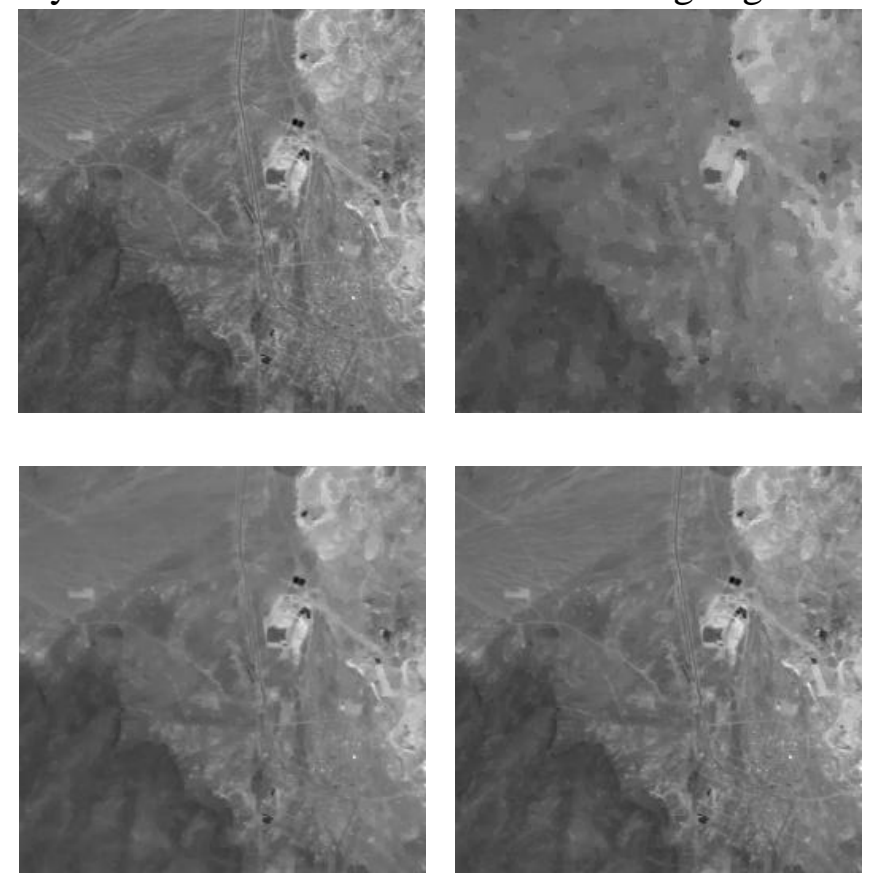

Fig. 2 The reconstructed second scene of Cuprite when the measurement rate is 0.2 (upper left: the original imageof the 80-th spectral band, upper right: reconstructed by $l_{1}$, bottom left: reconstructed by MT-BCS, bottom right: reconstructed by the proposed method) 


\section{Conclusions}

In this paper, we proposed an improved algorithm for the reconstruction of hyperspectral images in compressive whiskbroom imaging. In the acquisition of compressive measurements, spectral vector is separately acquired using compressive sampling, in the same way as actual satellite whiskbroom sensors operate. The reconstruction relies on the MT-BCS and a progressive refinement based on linear predictors to jointly process the measurements of each spectral vector, in order to exploit both spectral and spatial correlation at the same time. Experiments run on AVIRIS images show that the number of measurements required for the HSI reconstruction is significantly reduced comparing to the other conventional methods.

\section{Acknowledgements}

This work was supported by Guangdong Natural Science Foundation (2016A030307044, 2016A030307045, 2014A030307029), the Shaoguan City Scientific Program(441-99000311) and the Shaoguan University Scientific Program(314-140685).

\section{References}

[1] D.Donoho. Compressed sensing. IEEE Trans. On Information Theory, 2006, 52(2): 489-509.

[2] Jia Yingbiao, Feng Yan,Wang Zhongliang. Reconstructing Hyperspectral Images From Compressive Sensors via Exploiting Multiple Priors. Spectroscopy Letters.2015,48(1):22-26.

[3] R Willett, M F Duarte, M A Davenport, R G Baraniuk. Sparsity and Structure in Hyperspectral Imaging: Sensing, Reconstruction, and Target Detection. IEEE Signal Processing Magazine, 2014,31(1):116-126.

[4] J. E. Fowler. Compressive Pushbroom and Whiskbroom Sensing for Hyperspectral Remote-Sensing Imaging. in Proceedings of the International Conference on Image Processing, Paris, France, October 2014: 684-688.

[5] S Ji, D Dunson, L Carin. Multitask compressive sensing. IEEE Transactions on Signal Process, 2009, 57(1): 92-106.

[6] Shihao Ji, Ya Xue, Lawrence Carin. Bayesian Compressive Sensing. IEEE Transactions on Signal Processing,2008,56(6):2346-2356.

[7] Giulio Coluccia, Enrico Magli. A Novel Progressive Image Scanning and Reconstruction Scheme Based on Compressed Sensing and Linear Prediction. 13th IEEE International Conference on Multimedia and Expo(ICME), Melbourne, Australia, 2012: 866-871.

[8] Simeon Kamdem Kuiteing, Giulio Coluccia, Alessandro Barducci, Mauro Barni, Enrico Magli: Compressive hyperspectral imaging using progressive total variation. ICASSP 2014: 7794-7798.

[9] S Mun, J E Fowler. Motion-Compensated Compressed-Sensing Reconstruction for Dynamic MRI. in Proceedings of the International Conference on Image Processing, Melbourne, Australia, 2013:1006-1010. 